\section{DIN EN ISO 15195}

G. Schumann

Hannover, Deutschland

\section{Englischer Begriff ISO 15195}

Beschreibung Die Internationale Norm DIN EN ISO 15195 „Laboratoriumsmedizin - Anforderungen an die Referenzmesslaboratorien“, der ISO 17025 (๖ DIN EN ISO/IEC 17025 ) und ISO 9001 ( $\triangleright$ DIN EN ISO 9001) zugrunde liegen, enthält Anforderungen an die Qualität und Kompetenz von Referenzmesslaboratorien. Die Norm nimmt auf die besonderen Aspekte der Referenzlaboratorien, auch als Kalibrierlaboratorien bezeichnet, auf dem Gebiet der Laboratoriumsmedizin Bezug. Die von medizinischen Laboratorien erstellten Ergebnisse sollen auf Referenzmaterialien und/oder
Referenzmessverfahren höherer Ordnung, sofern verfügbar, rückführbar sein. Dadurch soll die Übereinstimmung von Messergebnissen unabhängig von Zeit und Ort der Messung erzielt werden. Die Norm ISO 15195 bildet die Grundlage für die Akkreditierung eines Referenzmessverfahrens für eine medizinische Messgröße.

Die Norm in der Fassung von 2003 muss im Technischen Komitee 212 (TC212) von ISO überarbeitet werden, um Änderungen zu berücksichtigen, die sich auf die normativen Verweise auf ISO 17025 ableiten. Die revidierte Norm ISO 15195 wird nicht vor 2018 verabschiedet sein.

\section{Literatur}

DIN EN ISO 15195 (2003) Laboratoriumsmedizin - Anforderungen an die Referenzmesslaboratorien. Beuth-Verlag, Berlin 